47th AIAA Aerospace Sciences Meeting

January 5-8 2009, Orlando, FL

\title{
Multi-Dimensional, Inviscid Flux Reconstruction for Simulation of Hypersonic Heating on Tetrahedral Grids
}

\author{
Peter A Gnoffo* \\ NASA Langley Research Center, Hampton, VA 23681-2199
}

\begin{abstract}
The quality of simulated hypersonic stagnation region heating on tetrahedral meshes is investigated by using a three-dimensional, upwind reconstruction algorithm for the inviscid flux vector. Two test problems are investigated: hypersonic flow over a three-dimensional cylinder with special attention to the uniformity of the solution in the spanwise direction and hypersonic flow over a three-dimensional sphere. The tetrahedral cells used in the simulation are derived from a structured grid where cell faces are bisected across the diagonal resulting in a consistent pattern of diagonals running in a biased direction across the otherwise symmetric domain. This grid is known to accentuate problems in both shock capturing and stagnation region heating encountered with conventional, quasi-onedimensional inviscid flux reconstruction algorithms. Therefore the test problem provides a sensitive test for algorithmic effects on heating. This investigation is believed to be unique in its focus on three-dimensional, rotated upwind schemes for the simulation of hypersonic heating on tetrahedral grids. This study attempts to fill the void left by the inability of conventional (quasi-one-dimensional) approaches to accurately simulate heating in a tetrahedral grid system. Results show significant improvement in spanwise uniformity of heating with some penalty of ringing at the captured shock. Issues with accuracy near the peak shear location are identified and require further study.
\end{abstract}

\section{Nomenclature}

Bold face, lowercase variable names refer to vectors. Bold face, uppercase variable names refer to matrices.

Roman symbols

A

$E$

$\mathbf{f}_{x^{\prime}}$

$H$

$\vec{i}, \vec{j}, \vec{k}$

$M W$

$\vec{n}_{x^{\prime}}$

$\vec{n}_{A 1}, \vec{n}_{A 2}, \vec{n}_{A 3}$

$p$

$\mathbf{q}$

$\tilde{\mathbf{q}}_{x^{\prime}}$

$\mathbf{R}$

$T$ area

total energy

flux in $x^{\prime}$ direction, $\left[\rho U_{x^{\prime}}, \rho u U_{x^{\prime}}+p n_{x, x^{\prime}}, \rho v U_{x^{\prime}}+p n_{y, x^{\prime}}, \rho w U_{x^{\prime}}+p n_{z, x^{\prime}}, \rho U_{x^{\prime}} H\right]^{T}$ total enthalpy

unit vectors in Cartesian coordinate directions $(x, y, z)$, respectively

molecular weight

unit vector in $x^{\prime}$ direction, $n_{x, x^{\prime}} \vec{i}+n_{y, x^{\prime}} \vec{j}+n_{z, x^{\prime}} \vec{k}$

unit vectors orthogonal to faces $A 1, A 2, A 3$

pressure

conserved variables $[\rho, \rho u, \rho v, \rho w, \rho E]^{T}$

characteristic variable in direction $x^{\prime}, d \tilde{\mathbf{q}}_{x^{\prime}}=\mathbf{R}_{x^{\prime}} d \mathbf{q}$

eigenvector matrix for flux Jacobian, $\frac{\partial \mathbf{f}_{x^{\prime}}}{\partial \mathbf{q}}=\mathbf{R}_{x^{\prime}}^{-1} \boldsymbol{\Lambda}_{x^{\prime}} \mathbf{R}_{x^{\prime}}$

temperature

*Senior Research Engineer, Aerothermodynamics Branch; AIAA Fellow 


\section{Roman symbols, continued}

$u, v, w$
$U_{x^{\prime}}$
$x^{\prime}, y^{\prime}, z^{\prime}$
Greek symbols
$\alpha$
$\beta$
$\Lambda$
$\rho$
$\Omega$
Subscripts
$1,2,3$
$1-2,1-3,2-3$
$A$
$k$
$L$
$L S q$
$n$
$R$
$x^{\prime}$

density
Cartesian velocity components

velocity component in $x^{\prime}$ direction, $u n_{x, x^{\prime}}+v n_{y, x^{\prime}}+w n_{z, x^{\prime}}$

principal directions across element

coefficient relative to face for defining derivative in an element, Eq. 30

coefficient relative to node for defining derivative in an element, Eq. 8

diagonal matrix of eigenvalues for flux Jacobian, $\frac{\partial \mathbf{f}_{x^{\prime}}}{\partial \mathbf{q}}=\mathbf{R}_{x^{\prime}}^{-1} \boldsymbol{\Lambda}_{x^{\prime}} \mathbf{R}_{x^{\prime}}$

element volume

node indices in triangular element

edge identification as function of node endpoints

element id, centroid of element

face oriented index defined by index of opposite node in the element

left virtual node in Option 1

computed by Least Squares of gradients in surrounding elements

node oriented index in element

right virtual node in Option 1

function of direction $x^{\prime}$, usually involving $U_{x^{\prime}}$

\section{Introduction}

The simulation of hypersonic flows with fully unstructured (tetrahedral) grids has severe problems with respect to the prediction of stagnation region heating. ${ }^{1,2}$ The problems arise for two reasons.

First, good shock capturing in the hypersonic regime requires alignment of the grid with the captured shock. Alignment here means that all surfaces of a control volume in contact with the shock are either parallel to the discontinuity or orthogonal to the discontinuity. Any skewness present in a conventional upwind scheme (in which first-order reconstruction is a function of only two nodes on opposite sides of a shared face) results in a smearing of the flow which manifests itself as a non-physical distribution of entropy from streamline to streamline crossing the shock. In the stagnation region, there is very little dissipation of these entropy gradients as they pass from the shock to the boundary-layer edge. Consequently, the surface heating is very sensitive to these non-physical gradients. Structured grid systems are generally easy to align with the captured bow shock. NASA's main computational aerothermodynamic simulation codes LAURA ${ }^{3,4}$ and DPLR ${ }^{5}$ both take great pains to align structured grid with the captured bow shock to improve solution quality but accept the limitation that internal shocks (e.g. wake recompression, control surface compression) are computed with no special grid consideration. (In fact, sharp double cone code validation test problems ${ }^{6}$ revealed that extremely fine structured grids were required to achieve a grid converged solution in a problem where structured grid could not be easily aligned with internally reflected shocks and shear layer over a separation bubble. ${ }^{7,8}$ ) Tetrahedral cell topologies by their very nature will always have at least one surface that is skewed to the captured shock.

Second, tetrahedral grids are not well suited to the preservation of symmetry and the biased tetrahedral grids used in the tests herein are particularly ill-suited for this purpose. Asymmetric elements tend to induce non-physical cross flows. Again, this problem is particularly evident in the stagnation region where the physical flow velocities themselves are small, the non-physical, grid-induced velocities are of corresponding magnitude, and the surface heating is a sensitive indicator of these problems. The combination of random jaggedness in the shock capturing process as well as non-physical cross flow velocities cross-couple to produce unacceptable surface heating predictions.

Problems with heating can be overcome if one uses semi-structured grid (e.g. prisms) across the boundary layer and adapts the grid to the shock. Nompelis et. al. ${ }^{9}$ show excellent heating results for the cylinder test problem ${ }^{10}$ on families of grids where a prismatic grid was adapted to the shock and acceptable heating results when an unbiased (random face orientation), tetrahedral grid was adapted to the shock. In this 
case, random jaggedness may disguise some issues with shock capturing on tetrahedral elements. Prismatic elements are relatively easy to generate on blunt body geometries and their superior performance with respect to aeroheating predictions have led to their use as standard practice in unstructured simulations. There is no better alternative with todays algorithms than to use prismatic elements to capture the boundary layer and bow shock. Note however that application of shock fitting in the context of unstructured grids may offer improved solution quality by bypassing the difficulties associated with capturing a strong bow shock. ${ }^{11}$

If one is doing a hypersonic simulation without any free shear layers or internal shocks the specialized application of prismatic elements at the body and bow shock is perfectly acceptable. If such flow structures exist - as they do in almost all interesting problems - then the accuracy of any algorithm must be questioned when it ignores special topological grid requirements where the features are harder to resolve.

A challenge problem was identified ${ }^{2,10}$ in which a highly biased, tetrahedral grid is applied to the simulation of a hypersonic flow over a $3 \mathrm{D}$ cylinder at $V_{\infty}=5000 \mathrm{~m} / \mathrm{s}, \rho_{\infty}=0.001 \mathrm{~kg} / \mathrm{m}^{3}$ and $T_{\infty}=200 \mathrm{~K}$ in air $\left(M_{\infty} \approx 17\right)$. (In fact, other hypersonic free stream conditions are acceptable - the challenge here is to recover the proper spanwise uniformity within 1\%). A successful simulation requires a constant spanwise simulation of heating, pressure, and shear and symmetric simulation of these quantities on the right and left sides of the cylinder. The grid bias accentuates any issues with the algorithm. To date there have been no successful simulations of this challenge problem on the given grid using conventional upwind formulations. A recent thesis on a related unstructured grid without bias (random orientation of tetrahedra in spanwise direction) produced excellent heating symmetry using a high-order Discontinuous Galerkin finite element method with a PDE based artificial viscosity. ${ }^{12}$

A flux reconstruction algorithm that is not constrained by the local orientation of the grid is thought to have greater potential for a successful resolution of the challenge problem. The finite element method noted above ${ }^{12}$ has an inherently three-dimensional stencil in the evaluation of flux. Other approaches using more conventional, upwind, finite-volume formulations are known as "rotated upwind schemes". These were originally generated to address problems in capturing shocks on structured grids that were oblique to the grid. ${ }^{13,14}$ The underlying ideas for this class of algorithm have been adapted for use in three-dimensional reconstruction using the node-based unstructured solver FUN3D and are applied to the challenge problem.

\section{Multi-Dimensional Reconstruction Algorithm}

\section{A. Control Volume}

A triangular grid system is presented in Fig. 1. The two-dimensional reconstruction algorithm is developed relative to this figure. The extension of the algorithm to three dimensions is presented in Appendix A.

Assume a node based scheme as illustrated in Fig. 1 with $(x, y)$ coordinates of all nodes $(1,2,3, \ldots)$ given and dependent variables $\mathbf{q}$ at these same nodes to be determined by solution of conservation laws. The conservation laws are discretized relative to the dual control volume, represented by dashed lines in Fig. 1. The dual volume around node 3 passes through the centroids of all elements surrounding the node $(A, B, C, D, E, F)$ and the midpoints of all edges separating these elements.

\section{B. Reconstruction Overview - Principal (Rotated) Direction}

Consider a conventional, quasi-one-dimensional, first-order reconstruction of inviscid flux across face $A c B$ separating the dual volumes around nodes 1 and 3 . This flux is defined as a function of information at nodes 1 and 3 of edge $c$ :

$$
\mathbf{f}_{A c B}=\frac{1}{2}\left[\mathbf{f}_{3}+\mathbf{f}_{1}-\mathbf{R}_{c}\left|\boldsymbol{\Lambda}_{c}\right| \mathbf{R}_{c}^{-1}\left(\mathbf{q}_{3}-\mathbf{q}_{1}\right)\right]
$$

Inviscid fluxes are then defined using a loop over edges and the reconstruction direction is fully determined by the grid.

The basic idea in multi-dimensional reconstruction is that: (1) reconstruction directions should be based on local flow characteristics and not be constrained by the grid; and (2) the flux components for any face will utilize a stencil employing all nodes of a surrounding element. Whereas node based schemes loop over edges when using quasi-one-dimensional reconstruction the multi-dimensional reconstruction focuses on a loop over

elements. Consider the two-dimensional element defined by nodes $(1,2,3)$ with centroid $A$ in Fig. 1 . The computation of inviscid flux in this element employs the same infrastructure for computing viscous flux. 


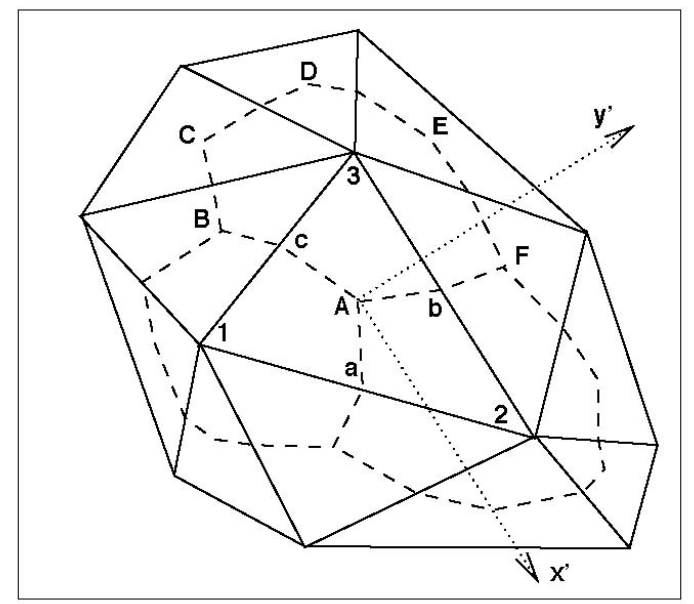

Figure 1. Schematic of node-based, unstructured grid system showing element centroids (uppercase letters), edge midpoints (lowercase letters), nodes (numerals), the dual volume (dashed lines), and the principal directions (dotted lines with arrowhead).

A principal direction in the element, $x^{\prime}$, is defined by the direction of $\vec{\nabla} \rho$, computed using the same Green Gauss evaluation of the derivatives as applied for the viscous terms. If density is locally constant the principal direction aligns with the local velocity computed as the average of nodal values. Inviscid flux within the element will be computed along and orthogonal to the principal flow direction.

The flux components are defined using nodal weights based on the Green-Gauss formulation for derivatives in the component directions. Thus,

$$
\frac{\partial \mathbf{f}_{A}}{\partial x^{\prime}}=\frac{1}{\Omega_{A}} \sum_{k=\text { faces }} \overline{\mathbf{f}}_{k} A_{k} n_{k, x^{\prime}}
$$

where $\overline{\mathbf{f}}_{k}$ is the average value of $\mathbf{f}$ across all nodes defining surface $k$ of element $A$. With reference to Fig. 1 this derivative can be written

$$
\frac{\partial \mathbf{f}_{x^{\prime}, A}}{\partial x^{\prime}}=\alpha_{3, x^{\prime}}\left(\mathbf{f}_{2, x^{\prime}}+\mathbf{f}_{1, x^{\prime}}\right)+\alpha_{1, x^{\prime}}\left(\mathbf{f}_{3, x^{\prime}}+\mathbf{f}_{2, x^{\prime}}\right)+\alpha_{2, x^{\prime}}\left(\mathbf{f}_{1, x^{\prime}}+\mathbf{f}_{3, x^{\prime}}\right)
$$

where

$$
\alpha_{k, x^{\prime}}=\frac{A_{k} n_{k, x^{\prime}}}{2 \Omega_{A}}
$$

and $k$ is a face index identified by the number of the opposite node. The sum $\sum_{k=\text { faces }} \alpha_{k, x^{\prime}}=0$ for any closed element. It is convenient to reorder and scale the coefficients as follows.

$$
\begin{aligned}
\frac{\partial \mathbf{f}_{x^{\prime}, A}}{\partial x^{\prime}} & =\left(\alpha_{2, x^{\prime}}+\alpha_{3, x^{\prime}}\right) \mathbf{f}_{1}+\left(\alpha_{3, x^{\prime}}+\alpha_{1, x^{\prime}}\right) \mathbf{f}_{2}+\left(\alpha_{1, x^{\prime}}+\alpha_{2, x^{\prime}}\right) \mathbf{f}_{3} \\
& =\frac{1}{\Delta x^{\prime}}\left[\beta_{1, x^{\prime}} \mathbf{f}_{1}+\beta_{2, x^{\prime}} \mathbf{f}_{2}+\beta_{3, x^{\prime}} \mathbf{f}_{3}\right]
\end{aligned}
$$

Note that $\Delta x^{\prime}$ can be interpreted as a distance across the element in $x^{\prime}$ direction. It is defined

$$
\frac{1}{\Delta x^{\prime}}=\sum_{n=\text { nodes }}\left(\left|\alpha_{n-1, x^{\prime}}+\alpha_{n+1, x^{\prime}}\right|\right)
$$

where a cyclic indexing is assumed. The reordering and scaling yield the following relations for $\beta_{n, x^{\prime}}$.

$$
\begin{aligned}
\beta_{n, x^{\prime}} & =\Delta x^{\prime}\left(\alpha_{n-1, x^{\prime}}+\alpha_{n+1, x^{\prime}}\right) \\
\sum_{n} \beta_{n, x^{\prime}} & =0 \\
\sum_{n}\left|\beta_{n, x^{\prime}}\right| & =1
\end{aligned}
$$


Two options for computing $\mathbf{f}_{x^{\prime}}$ are developed. Both mirror the baseline quasi-one-dimension reconstruction using Roe's averaging across nodes. The first option averages dependent variables at two virtual nodes used in the reconstruction. The second option utilizes actual nodes and applies a weighted average to the computed flux on surrounding edges.

\section{Option 1 - Virtual Node Averaging}

A right and left virtual state for computing $\mathbf{f}_{x^{\prime}}$ are computed as follows.

$$
\begin{gathered}
\mathbf{q}_{R, x^{\prime}}=\frac{1}{2} \sum_{n=n o d e s}\left(\left|\beta_{n, x^{\prime}}\right|+\beta_{n, x^{\prime}}\right) \mathbf{q}_{n} \\
\mathbf{q}_{L, x^{\prime}}=\frac{1}{2} \sum_{n=n o d e s}\left(\left|\beta_{n, x^{\prime}}\right|-\beta_{n, x^{\prime}}\right) \mathbf{q}_{n} \\
\mathbf{f}_{x^{\prime}}=\frac{1}{2}\left[\mathbf{f}(\mathbf{q})_{L, x^{\prime}}+\mathbf{f}(\mathbf{q})_{R, x^{\prime}}-\mathbf{R}_{x^{\prime}}^{-1}\left|\boldsymbol{\Lambda}_{x^{\prime}}\right|\left(d \tilde{\mathbf{q}}_{x^{\prime}}-d \tilde{\mathbf{q}}_{x^{\prime}, l i m}\right)\right]
\end{gathered}
$$

where the left and right states of $\mathbf{f}$ are functions of the left and right states of $\mathbf{q}$, respectively. The matrices $\mathbf{R}_{x^{\prime}}$ and $\boldsymbol{\Lambda}_{x^{\prime}}$ are computed as functions of the Roe's average of the right and left states.

$$
\begin{gathered}
d \tilde{\mathbf{q}}_{x^{\prime}}=\mathbf{R}_{x^{\prime}}\left(\mathbf{q}_{R, x^{\prime}}-\mathbf{q}_{L, x^{\prime}}\right) \\
=\mathbf{R}_{x^{\prime}} \sum_{n=\text { nodes }} \beta_{n, x^{\prime}} \mathbf{q}_{n} \\
d \tilde{\mathbf{q}}_{R, x^{\prime}}=\Delta x^{\prime} \mathbf{R}_{x^{\prime}} \sum_{n=\text { nodes }}\left(\left|\beta_{n, x^{\prime}}\right|+\beta_{n, x^{\prime}}\right) \nabla \mathbf{q}_{n, L S q} \\
d \tilde{\mathbf{q}}_{L, x^{\prime}}=\Delta x^{\prime} \mathbf{R}_{x^{\prime}} \sum_{n=n o d e s}\left(\left|\beta_{n, x^{\prime}}\right|-\beta_{n, x^{\prime}}\right) \nabla \mathbf{q}_{n, L S q} \\
d \tilde{\mathbf{q}}_{x^{\prime}, l i m}=\operatorname{minmod}\left[2 d \tilde{\mathbf{q}}_{R, x^{\prime}}, 2 d \tilde{\mathbf{q}}_{x^{\prime}}, 2 d \tilde{\mathbf{q}}_{L, x^{\prime}}, \frac{1}{2}\left(d \tilde{\mathbf{q}}_{R, x^{\prime}}+d \tilde{\mathbf{q}}_{L, x^{\prime}}\right)\right]
\end{gathered}
$$

\section{Option 2 - Weighted Average of Edges to Principal Node}

Select the largest of $\left|\beta_{n, x^{\prime}}\right|$ to identify the principal node for construction of $f_{x^{\prime}}$ and express it as a function of the remaining coefficients. Assume node 3 is the principal node. Then

$$
\begin{aligned}
d f_{x^{\prime}} & =\beta_{1, x^{\prime}} f_{1}+\beta_{2, x^{\prime}} f_{2}+\beta_{3, x^{\prime}} f_{3} \\
& =\beta_{1, x^{\prime}} f_{1}+\beta_{2, x^{\prime}} f_{2}-\left(\beta_{1, x^{\prime}}+\beta_{2, x^{\prime}}\right) f_{3} \\
& =\beta_{1, x^{\prime}}\left(f_{1}-f_{3}\right)+\beta_{2, x^{\prime}}\left(f_{2}-f_{3}\right)
\end{aligned}
$$

The reconstructed flux in the direction $x^{\prime}, \mathbf{f}_{x^{\prime}}$, is computed as a weighted average of surrounding edges. Furthermore, it is noted that if any of the surrounding edges are parallel to $x^{\prime}$ then the weight of that edge will equal 1 and the weight of the other edges will equal zero.

$$
\begin{gathered}
\mathbf{f}_{x^{\prime}}=\left|\beta_{1, x^{\prime}}\right| \mathbf{f}_{1-3, x^{\prime}}+\left|\beta_{2, x^{\prime}}\right| \mathbf{f}_{2-3, x^{\prime}} \\
\mathbf{f}_{1-3, x^{\prime}}=\frac{1}{2}\left[\mathbf{f}_{1, x^{\prime}}+\mathbf{f}_{3, x^{\prime}}-\operatorname{sign}\left(\beta_{1, x^{\prime}}\right) \mathbf{R}_{1-3, x^{\prime}}^{-1}\left|\boldsymbol{\Lambda}_{1-3, x^{\prime}}\right|\left(d \tilde{\mathbf{q}}_{1-3, x^{\prime}}-d \tilde{\mathbf{q}}_{1-3, x^{\prime}, l i m}\right)\right] \\
\mathbf{f}_{2-3, x^{\prime}}=\frac{1}{2}\left[\mathbf{f}_{2, x^{\prime}}+\mathbf{f}_{3, x^{\prime}}-\operatorname{sign}\left(\beta_{2, x^{\prime}}\right) \mathbf{R}_{2-3, x^{\prime}}^{-1}\left|\boldsymbol{\Lambda}_{2-3, x^{\prime}}\right|\left(d \tilde{\mathbf{q}}_{2-3, x^{\prime}}-d \tilde{\mathbf{q}}_{2-3, x^{\prime}, l i m}\right)\right]
\end{gathered}
$$

where

$$
\begin{aligned}
d \tilde{\mathbf{q}}_{1-3, x^{\prime}} & =\mathbf{R}_{1-3, x^{\prime}}\left(\mathbf{q}_{1}-\mathbf{q}_{3}\right) \\
d \tilde{\mathbf{q}}_{2-3, x^{\prime}} & =\mathbf{R}_{2-3, x^{\prime}}\left(\mathbf{q}_{2}-\mathbf{q}_{3}\right) \\
d \tilde{\mathbf{q}}_{1-3, x^{\prime}, l i m} & =\operatorname{minmod}\left[2 d \tilde{\mathbf{q}}_{1, x^{\prime}}, 2 d \tilde{\mathbf{q}}_{1-3, x^{\prime}}, 2 d \tilde{\mathbf{q}}_{3, x^{\prime}}, \frac{1}{2}\left(d \tilde{\mathbf{q}}_{1, x^{\prime}}+d \tilde{\mathbf{q}}_{3, x^{\prime}}\right)\right] \\
d \tilde{\mathbf{q}}_{2-3, x^{\prime}, l i m} & =\operatorname{minmod}\left[2 d \tilde{\mathbf{q}}_{2, x^{\prime}}, 2 d \tilde{\mathbf{q}}_{2-3, x^{\prime}}, 2 d \tilde{\mathbf{q}}_{3, x^{\prime}}, \frac{1}{2}\left(d \tilde{\mathbf{q}}_{2, x^{\prime}}+d \tilde{\mathbf{q}}_{3, x^{\prime}}\right)\right] \\
d \tilde{\mathbf{q}}_{n, x^{\prime}} & =\Delta x^{\prime} \mathbf{R}_{n, x^{\prime}} \nabla \mathbf{q}_{n, L S q} \overrightarrow{\mathbf{n}}_{x^{\prime}}
\end{aligned}
$$




\section{E. Component Assembly on Faces}

Both option 1 (Eq. 13) and option 2 (Eq. 19) present algorithms to define the flux, $\mathbf{f}_{x^{\prime}}$, in the principal direction $x^{\prime}$. The same logic is applied to construct $\mathbf{f}_{y^{\prime}}$. Within each two-dimensional, triangular element there are three partial faces. The partial faces $A c$ and $A b$ can be combined into an equivalent face $A 3$ separating node 3 from the other nodes in the element. In like manner $A c$ and $A a$ form $A 1$ and $A a$ and $A b$ form $A 2$. The flux across each equivalent face is constructed from the component flux in the principal direction and orthogonal to the principal direction. Thus, for element with centroid $A$ as shown in Fig. 1 the flux through each equivalent face is

$$
\mathbf{f}_{A n}=n_{A n, x^{\prime}} \mathbf{f}_{x^{\prime}}+n_{A n, y^{\prime}} \mathbf{f}_{y^{\prime}}
$$

where $A n$ refers to face separating node $n$ from other nodes in the element.

\section{F. Shock Capturing}

Both options 1 and 2 are observed to admit temperature undershoots in capturing strong bow shocks. This undershoot occurs for strong shocks even in the case of a first-order reconstruction where $d \tilde{\mathbf{q}}_{x^{\prime}, l i m}=0$. Though every component of the reconstructed flux uses a baseline Total Variation Diminishing (TVD) algorithm the net formulation is not TVD. A floor on temperature is specified, but this practice causes the convergence to stall as temperatures are reset. Modifying the viscosity within the shock layer to produce a cell Reynolds number equal to 8 has been observed to help this problem at the expense of a thicker captured shock. The algorithm is still being tested. Preliminary results indicate improvement in solution quality across the bow shock but degradation of solution quality in vicinity of shock / boundary-layer interactions.

\section{G. Accuracy}

Tests to evaluate order of accuracy for heating and shear on sequentially refined tetrahedral grids have been impeded by an inability to converge to a sufficiently low error norm. Issues with shock capturing noted above are believed to be the primary cause of the stalled convergence. A related issue is a ringing in the computation of the principal direction - a slight change in the computed density gradient direction effects the reconstruction. This ringing is easily suppressed by freezing the principal direction when the solution is converged below a user specified norm.

The concern here is that the midpoint of the virtual nodes does not in general coincide with the centroid of the element; consequently, the reconstructed flux is offset from the dual volume surface by some small distance within the element. The blunt body solutions that follow match the pressure and shock shape of second-order structured grid benchmarks. However, there are differences in the shear and heating relative to structured grid benchmarks that are larger than expected for a second-order algorithm. Algorithms to create a second-order correction to the reconstructed flux that places it exactly at the centroid have not yielded any significant improvement. In fact, these "corrections" destroy the symmetry that is the focus of the test problems which follow. Thus, the current algorithm should be considered a work in progress. It offers significant improvement over the baseline quasi-one-dimensional reconstruction algorithm for hypersonic flow simulation on tetrahedral grids but there are still accuracy issues in the simulation of shear and heating.

\section{Numerical Results}

\section{A. FUN3D}

The three-dimensional reconstruction scheme is tested within FUN3D, which is a node based, fully unstructured, finite volume solver of the Euler and Navier-Stokes equations. ${ }^{15}$ The method uses Least Squares (LS) gradient information to execute second-order accurate inviscid flux reconstruction. ${ }^{16}$ Viscous gradients are computed from a Green-Gauss formulation. A suite of modules includes all of the gas physics models in LAURA and VULCAN ${ }^{17}$ for thermodynamics, transport properties, chemical kinetics, and thermal relaxation. The baseline inviscid flux reconstruction algorithms utilize quasi-one-dimensional (edge-based) reconstruction using Roe's averaging with Yee's symmetric total variation diminishing algorithm adapted for unstructured grids. 


\section{B. Challenge Problem - Biased, Tetrahedral Grid on 3D Cylinder}

Accurate simulation of stagnation region heating in hypersonic flows is a key requirement for acceptance of any algorithm proposed for use in aerothermodynamic analyses. A structured grid solution generated with LAURA is used as both a benchmark and to generate initial grids for use in FUN3D as described originally in an earlier paper. ${ }^{2}$ The test problem ${ }^{10,18}$ uses $V_{\infty}=5000 \mathrm{~m} / \mathrm{s}, \rho_{\infty}=0.001 \mathrm{~kg} / \mathrm{m}^{3}$, and $T_{\infty}=200 \mathrm{~K}$. Sutherland's law for air is used to define transport properties in all perfect-gas cases. As noted previously, this simple problem provides insight into the ability of a scheme to cleanly capture the bow shock, smoothly resolve the post-shock stagnation region flow and predict a smoothly varying heating distribution around the stagnation point. These flowfield characteristics are particularly sensitive to the inviscid flux reconstruction algorithm and problems that are not evident in well aligned, structured (hexahedral) grids are exposed in the unstructured (tetrahedral) environment. ${ }^{18}$

The structured grid, adapted to the shock and boundary layer, from LAURA is converted from hexahedral elements to tetrahedral elements by adding diagonal edges consistently from minimum index to maximum index corners. A comparison of the grids in a plane of nodes perpendicular to the cylinder axis is presented in Fig. 2. The structured grid has 65 nodes from the body to the inflow boundary ahead of the bow shock and 61 nodes from the left to right outflow boundaries. Node placement is identical between the two grids. The placement of additional edges in the unstructured grid is the only difference. The strong biasing of diagonals in the grid is an intentional characteristic to expose algorithm deficiencies that may otherwise be averaged out in the simulation.

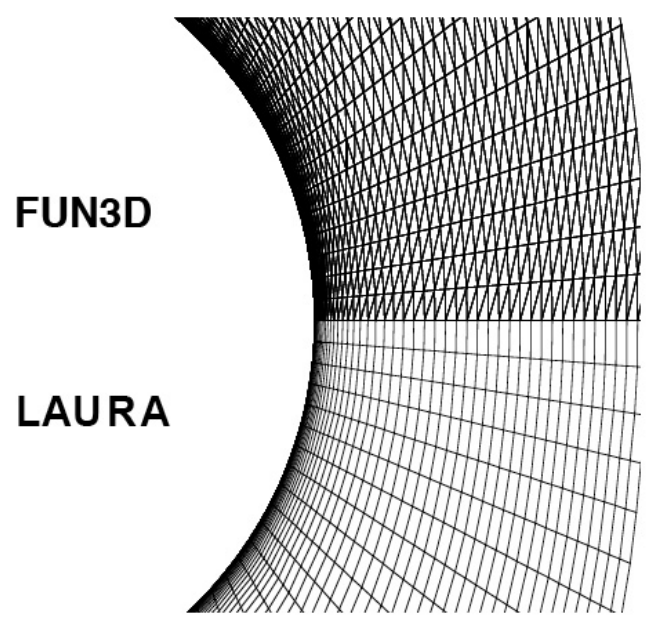

Figure 2. Structured grid (LAURA) and biased, unstructured grid (FUN3D) in plane orthogonal to cylinder surface.

A key element of this test problem is the addition of ten spanwise cells, shown in Fig. 3, across the cylinder, providing additional degrees of freedom in the simulation to allow asymmetries to develop. Earlier tests $^{18}$ (when the code was referred to as High Energy Flow Solver Synthesis - HEFSS) have shown that the single spanwise cell grids show good agreement with the structured code results in heating. (Fig. 4). The spanwise degrees of freedom enable non-physical cross-flow velocities to develop and irregular shock capture in the spanwise direction that combine to corrupt the predicted heating distribution.

\section{Structured Grid Reference Results}

Results from a previous paper ${ }^{2}$ are briefly repeated here to show the symmetry qualities being sought in the unstructured formulation. Structured, hexahedral grids are assessed in FUN3D to confirm that spanwise and circumferential symmetry is preserved. A perfect gas case $\left(\gamma=5 / 3, M W=2 M_{\infty}=4.25\right)$ is tested. A constant spanwise value of heating and shear is recovered when eigenvalue limiting is engaged as shown in Fig. 5. The near perfect overplotting of symbols in Fig. 5(a) for each of 11 spanwise nodes (ten spanwise 


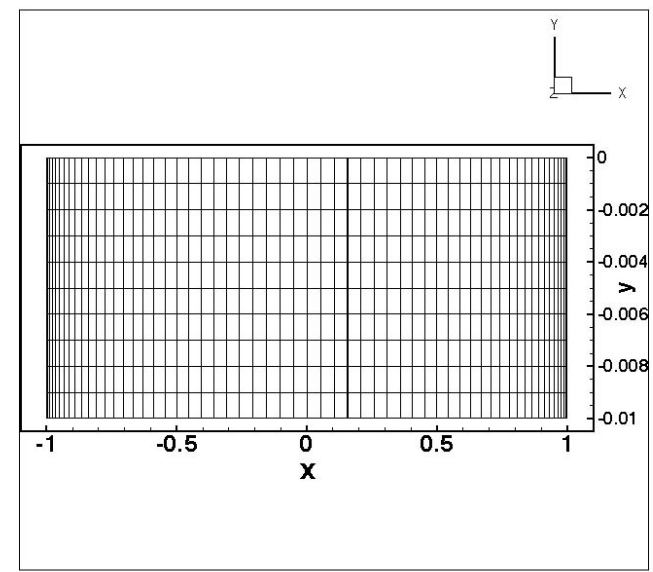

(a) Structured, quadrilateral surface elements

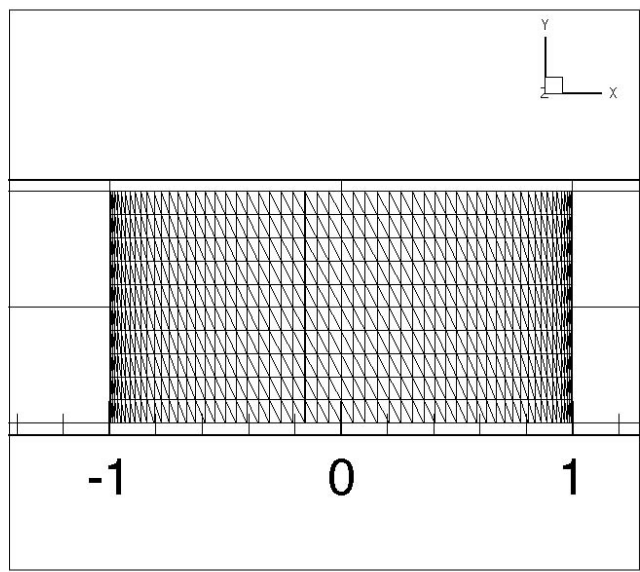

(b) Unstructured, triangular surface elements

Figure 3. Surface mesh on cylinder with ten rows of spanwise cells.

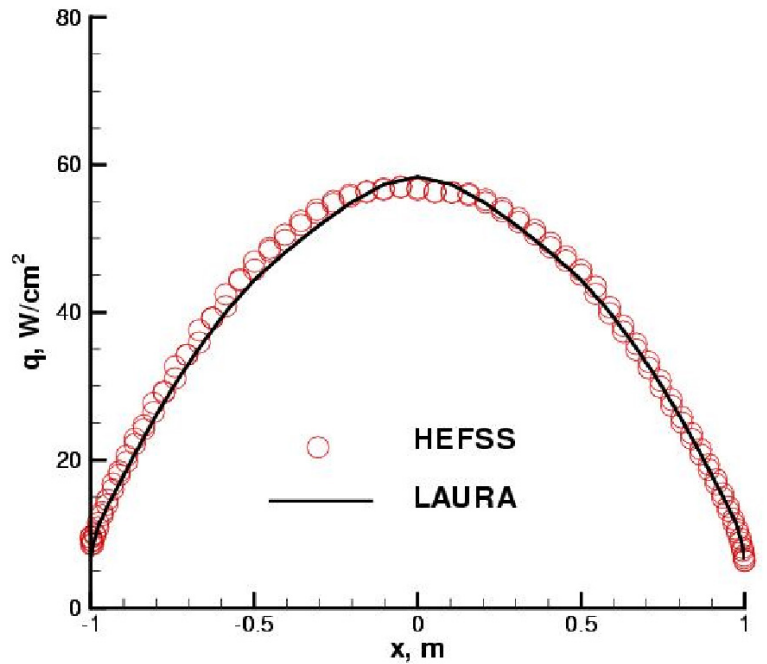

Figure 4. Surface heating over cylinder at standard test conditions with 5-species reacting air model and fully catalytic wall.

cells) at each $x$ location around the cylinder indicates that the baseline algorithm is behaving as expected for the structured grid case and provide a metric by which to evaluate the unstructured results.

\section{Conventional Upwind Results on Biased, Tetrahedral Grid}

The perfect gas test case described above was repeated on the equivalent unstructured grid with identical location of nodes. The structured and unstructured results for heating and shear were compared in an earlier paper $^{2}$ where an approximate spanwise variation of $\pm 10 \%$ about the mean was observed in peak values of heating and shear. There was also approximately $10 \%$ difference in the peak values of shear on the right and left sides of the cylinder for the unstructured grid. The large value of $\gamma=5 / 3$ and low molecular weight $M W=2$ produce a relatively large sound speed and moderate supersonic Mach number $M_{\infty}=4.25$. Even for this relatively low supersonic Mach number heating and shear asymmetries were produced on the challenge grid.

Selecting a perfect gas with $\gamma=7 / 5$ and $M W=28.8$ yields a lower sound speed and higher free stream 


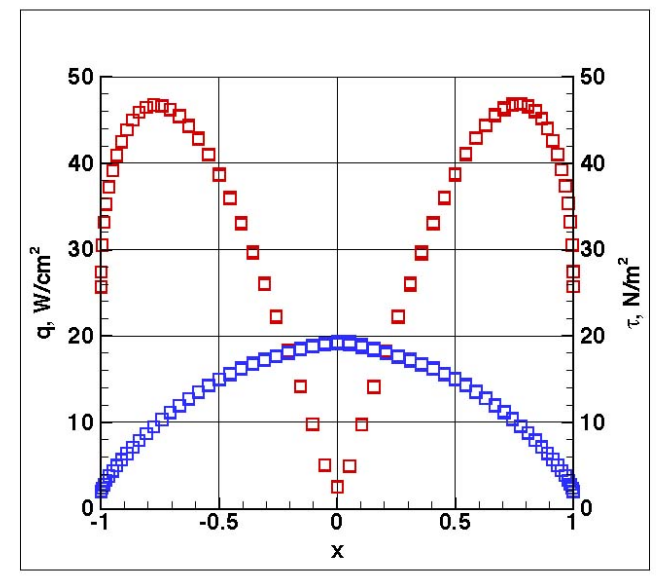

(a) Heating (blue squares) and shear (red squares) distribution

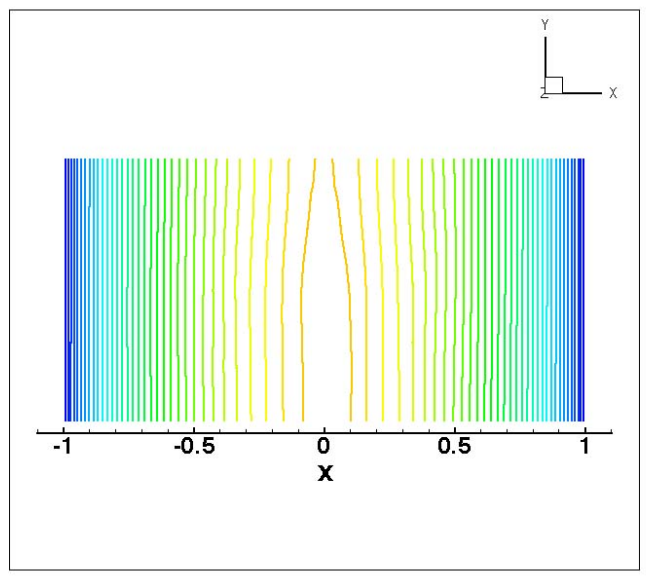

(b) Heating contours

Figure 5. Results on cylinder computed on structured grid for $\gamma=5 / 3$ and $M W=2$ with ten spanwise cells.

Mach number $M_{\infty}=17.34$. This more severe condition produces even larger spanwise variation in heating and shear (Fig. 6(a)) with $\pm 30 \%$ about the mean - an unacceptable result for aerothermodynamic analyses. Recall that the symbols at every $\mathrm{x}$ location should overplot and the peak value of shear on the right should equal the peak value of shear on the left. The LAURA result in this case is $52 \mathrm{~W} / \mathrm{cm}^{2}$ which is in good agreement with the spanwise mean of the heating at the stagnation point. The surface heating contours in Fig. 6(b) indicate that a slight drift in the velocity - probably induced by grid bias - produces a higher heating toward the front $(y=0)$ plane.

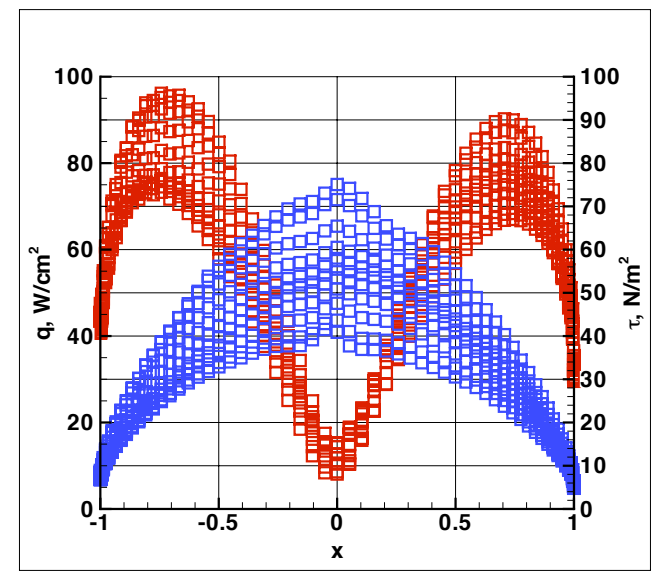

(a) Heating (blue squares) and shear (red squares) distribution for each of ten rows of spanwise cells.

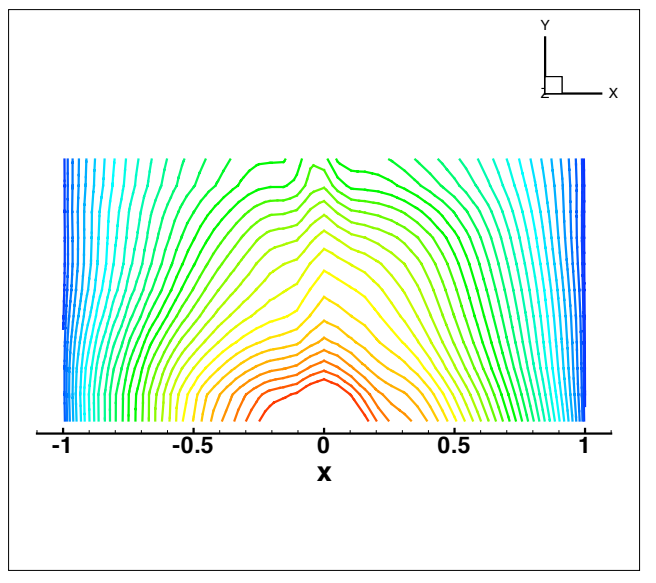

(b) Surface heating contours.

Figure 6. Simulation results for $\gamma=7 / 5$ and $M W=28.8$ using conventional reconstruction on the challenge grid with ten spanwise cells.

\section{Option 1 Results on Biased, Tetrahedral Grid}

Option 1 results for the $\gamma=7 / 5$ and $M W=28.8$ conditions as in the previous section are shown in Fig. 7 . The Option 1 results are presented as symbols and a benchmark, grid-converged, structured grid solution 
(LAURA) for the same case are presented as solid lines. The heating contours show greatly improved spanwise uniformity in Fig. 7(b) in the interior. Some end effects are evident at the top and bottom boundaries. The stagnation region heating is in good agreement with the benchmark but the distribution is flatter and the magnitude exceeds the benchmark by approximately $25 \%$ at the peak shear location. The shear contours in Fig. 7(d) show good spanwise uniformity as well. However, peak shear exceeds the benchmark value by approximately $20 \%$. If nodes near the span boundaries are eliminated from consideration, the spanwise variation is nearly zero in every surface quantity as shown in Fig. 7(c).

The cause of differences between the Option 1 algorithm and the benchmark are unknown at this time. Work is ongoing to resolve this difference and additional comments regarding this disparity will be offered in the Summary of Numerical Results section.

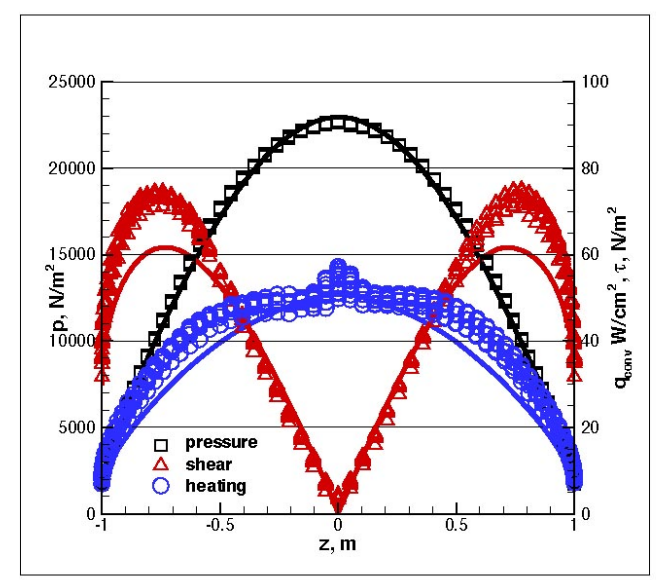

(a) Values of heating - blue circles, shear - red triangles, and pressure - black squares at nodes on surface. Figure shows results for all 11 surface nodes at a given $\mathrm{x}$ location.

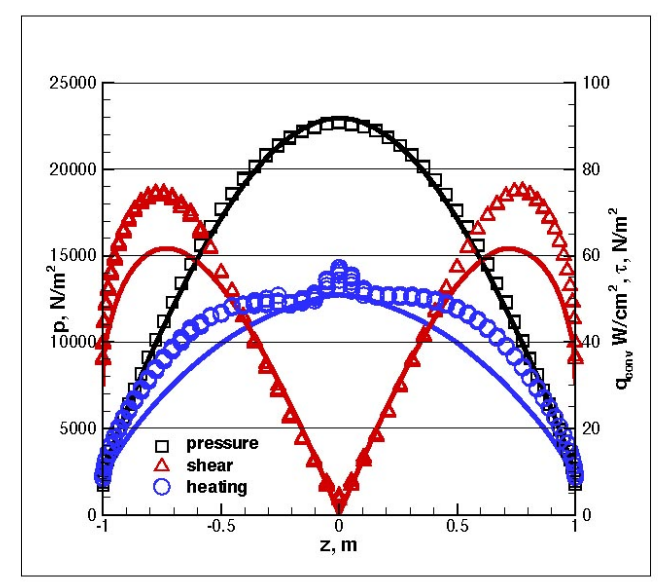

(c) Values of heating - blue circles, shear - red triangles, and pressure - black squares at nodes on surface. Figure shows results for 7 interior surface nodes at a given $\mathrm{x}$ location to remove edge effects.

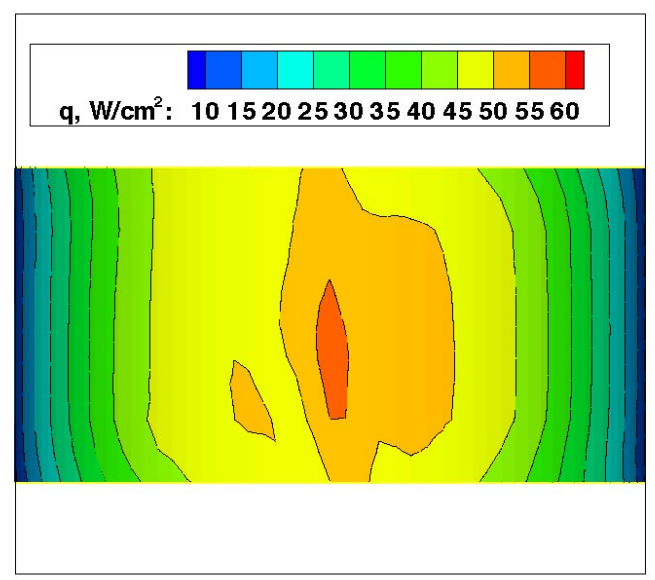

(b) Heating contours on surface showing significant improvement in spanwise symmetry. Some edge effects at spanwise boundaries still persist.

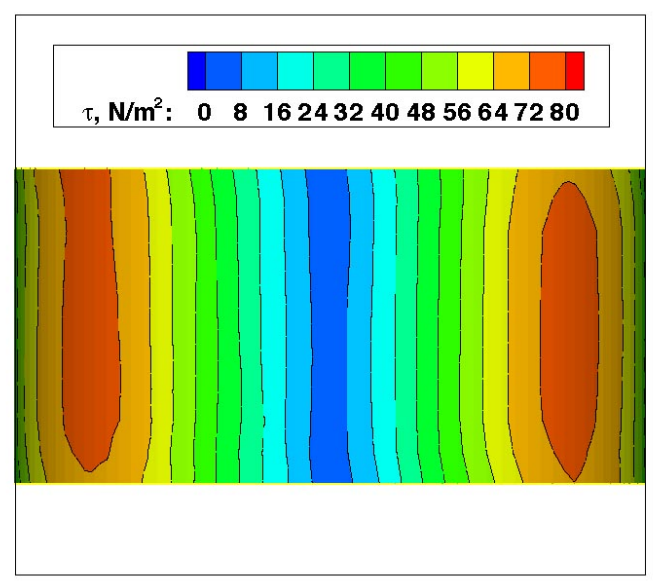

(d) Shear contours on surface showing interior spanwise symmetry.

Figure 7. Simulation results for $\gamma=7 / 5$ and $M W=28.8$ using Option 1 reconstruction on the challenge grid with ten spanwise cells. 


\section{Option 2 Results on Biased, Tetrahedral Grid}

Option 2 results on the test problems do not show any significant difference in solution quality. Even though each individual flux component was constructed from nodal values using the STVD formulation (without any intermediate averaging steps on primitive or conserved variables to virtual nodes as in Option 1) the net integrated flux around the surface of the control volume would still admit undershoots in temperature across the captured bow shock. The operation count for Option 2 is more expensive than for Option 1; consequently, without any improvement in solution quality Option 2 is not developed any further herein.

\section{Challenge Problem - Biased, Tetrahedral Grid on 3D Sphere}

The sphere test problem ${ }^{1}$ uses $V_{\infty}=4167 \mathrm{~m} / \mathrm{s}, \rho_{\infty}=0.0216 \mathrm{~kg} / \mathrm{m}^{3}, T_{\infty}=300 \mathrm{~K}$, and $T_{\text {wall }}=800 \mathrm{~K}$. Three sets of figures are presented to illustrate solution quality using the baseline, quasi-one-dimensional reconstruction on hexahedral and tetrahedral grids and using the multi-dimensional reconstruction (Option 1) on tetrahedral grids. The three sets include surface pressure in Fig. 8, surface heating in Fig. 9, and surface shear in Fig. 10. A close-up view of the hexahedral surface grid and tetrahedral surface grid are presented in Fig. 11.

The three sub-figures on the left of Fig. 8 from top to bottom show that surface pressure contours are consistently predicted on both hexahedral and tetrahedral grid systems with either reconstruction algorithm. The axisymmetry is well maintained with all contour lines appearing as concentric circles. On the right side of the figure an axisymmetric solution generated with the structured grid code LAURA is used as a benchmark shown as a solid black line. The symbols indicate the 3D unstructured solution cuts at 0 degrees (along the $\mathrm{x}$-axis) and at 45 degrees so that slices cut the surface grid at two different angles. The cut lines repeat across the axis. In the case of perfect symmetry, all symbols lie on top of each other. In the case of imperfect symmetry two sets of blue squares and two sets of red squares may be observed at any radial location. The three sub-figures on the right indicate good agreement with the structured grid benchmark and nearly perfect overplotting indicating excellent symmetry. Surface pressure is well predicted with every permutation of grid and reconstruction algorithm tested here. This result is consistent with results obtained on the spanwise cylinder test.

The solution quality for heating and shear are quite different than for pressure. The three sub-figures on the left of Fig. 9 show that the predicted heating in the stagnation region is sensitive to local grid orientation even in the case of a hexahedral grid system (Fig. 9(a)). A cross shaped contour pattern is formed in the stagnation region which transitions to concentric circles at approximately $40 \%$ of the maximum radius. (Fig. 9(b)) The standard reconstruction on the tetrahedral grids produces an even worse heating pattern as judged by lack of symmetry (Fig. 9(c)) and comparison to the benchmark solution in Fig. 9(d). These results are consistent with previous investigations. The Option 1 results with $3 \mathrm{D}$ reconstruction recover significant improvement in symmetry. A jag in the heating contours persists on the left side of Fig. 9(e) associated with a discontinuous change in the formation of the diagonal from the original hexahedral grid. Comparison with the benchmark solution at the stagnation point is improved compared to the $1 \mathrm{D}$ reconstruction on both the hexahedral and tetrahedral grids (Fig. 9(f)). However, the heating level drops below the benchmark beyond the $60 \%$ location of maximum radius. Note that the hexahedral grid system retains excellent comparison with the benchmark across this same range (Fig. 9(b)).

The quality of the predicted shear follows much the same pattern as the quality of the predicted heating as measured by symmetry (concentric circular contours) and comparisons to the benchmark. The hexahedral results show good symmetry and compare well with the benchmark (Figs. 10(a) and 10(b)); however, shear goes to zero at the stagnation point so it is not a sensitive indicator of solution quality in the stagnation region. The $1 \mathrm{D}$ reconstruction on tetrahedral grids provides a very poor simulation of shear by metrics of symmetry and difference from the benchmark. (Figs. 10(c) - 10(d)). The 3D reconstruction shows good symmetry but overpredicts the peak shear by approximately $8 \%$ relative to the benchmark. The shear peaks later than the benchmark and remains larger than the benchmark values beyond $70 \%$ of the maximum radius.

Two cuts are made through the stagnation streamline of the shock layer at 0 degrees and 45 degrees to show temperature contours (Fig. 12). Temperature contours from the 1D reconstruction (Fig. 12(a)) do not overlay in the stagnation region but otherwise show expected symmetry. The temperature contours from the 3D reconstruction (Fig. 12(b)) show excellent symmetry even in the stagnation region. 


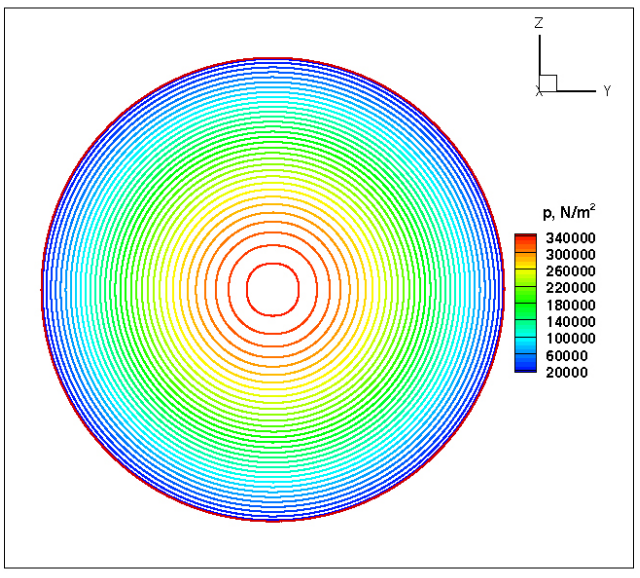

(a) Hex Grid, Surface Contours

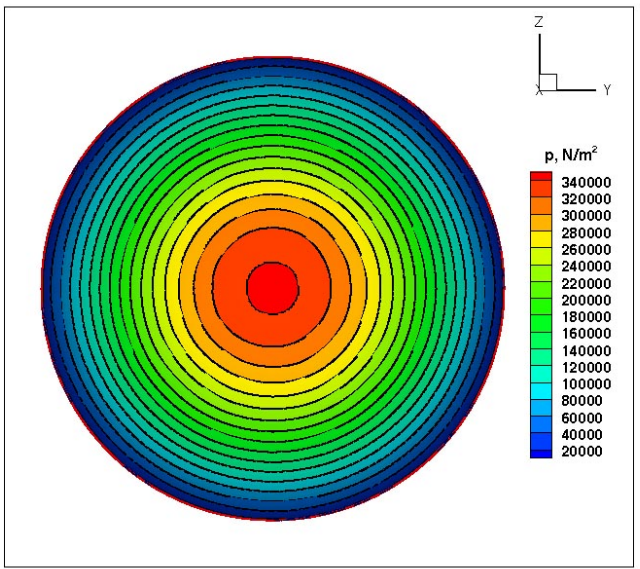

(c) Tet Grid, 1D reconstruction, Surface Contours

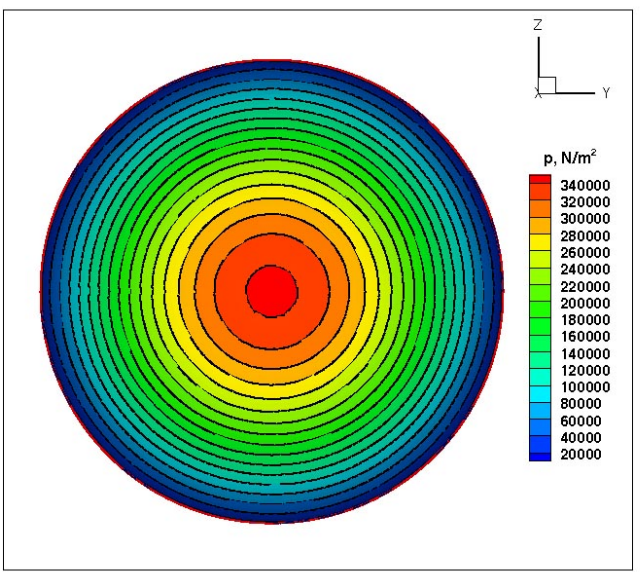

(e) Tet Grid, 3D reconstruction, Surface Contours

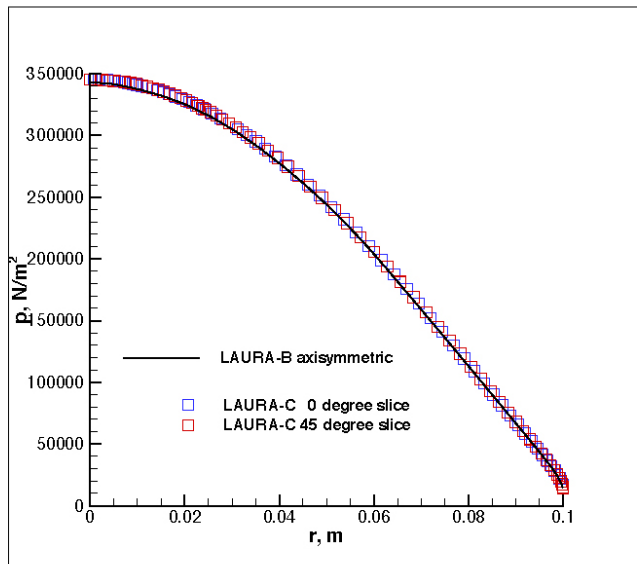

(b) Hex Grid, Line Cuts at 0 and 45 degrees

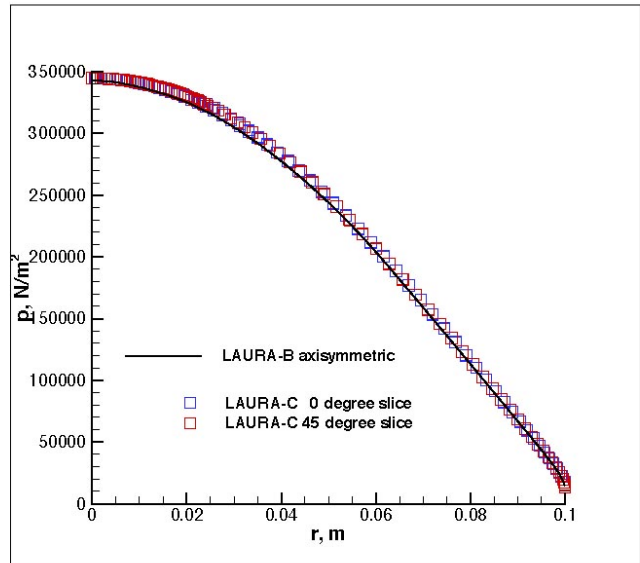

(d) Tet Grid, 1D reconstruction, Line Cuts at 0 and 45 degrees

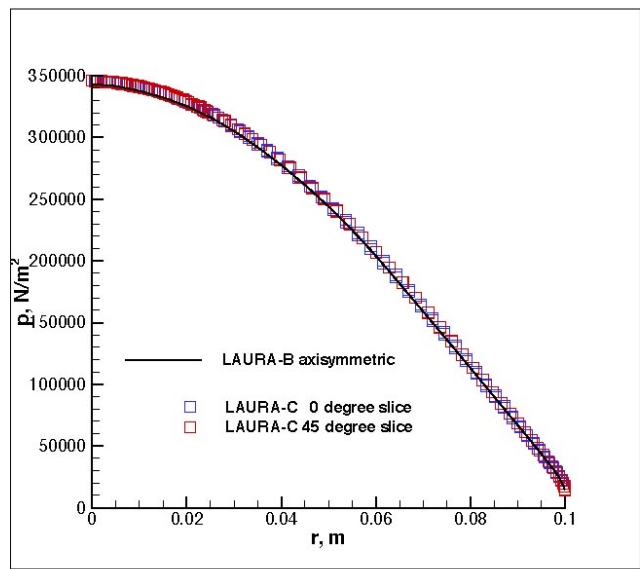

(f) Tet Grid, 3D reconstruction, Line Cuts at 0 and 45 degrees

Figure 8. Surface pressure on sphere test problem. 


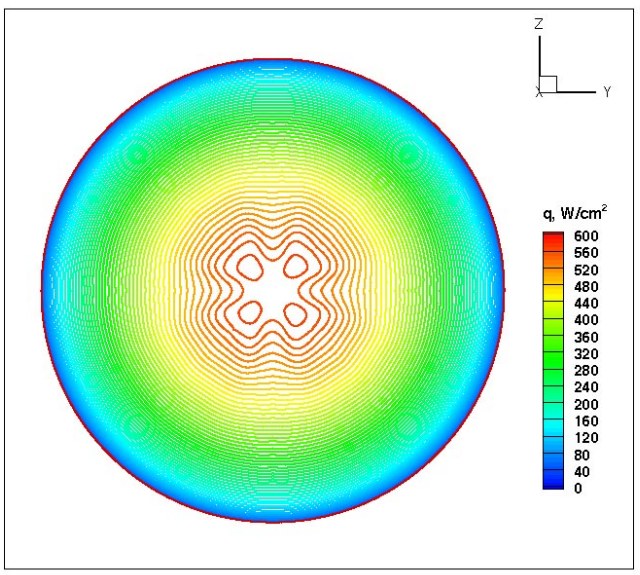

(a) Hex Grid, Surface Contours

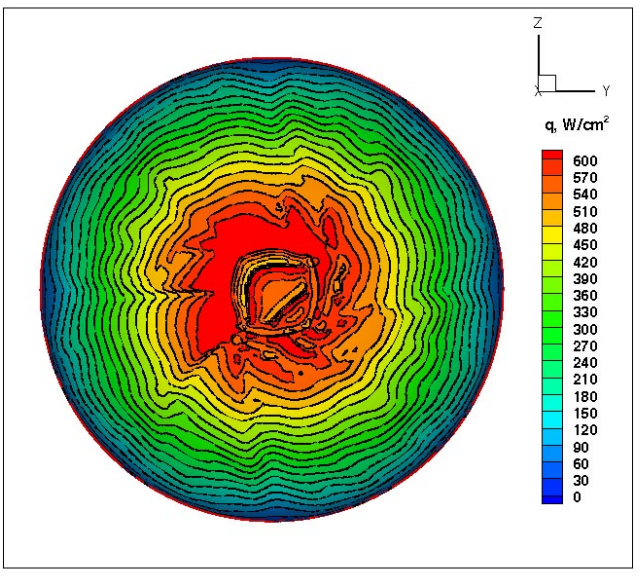

(c) Tet Grid, 1D reconstruction, Surface Contours

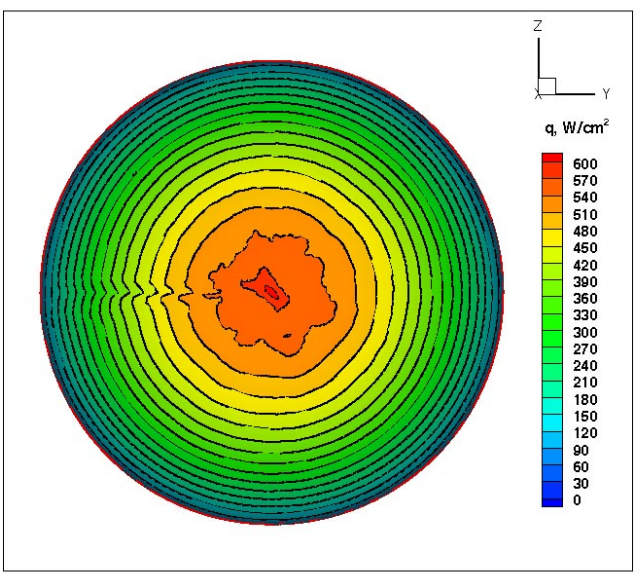

(e) Tet Grid, 3D reconstruction, Surface Contours

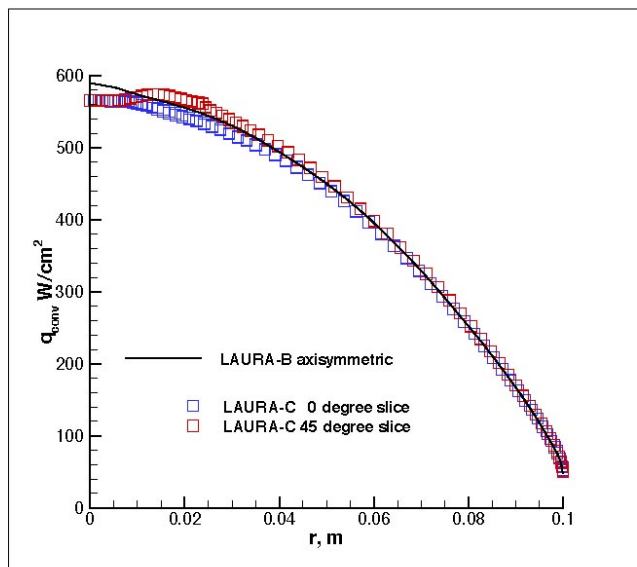

(b) Hex Grid, Line Cuts at 0 and 45 degrees

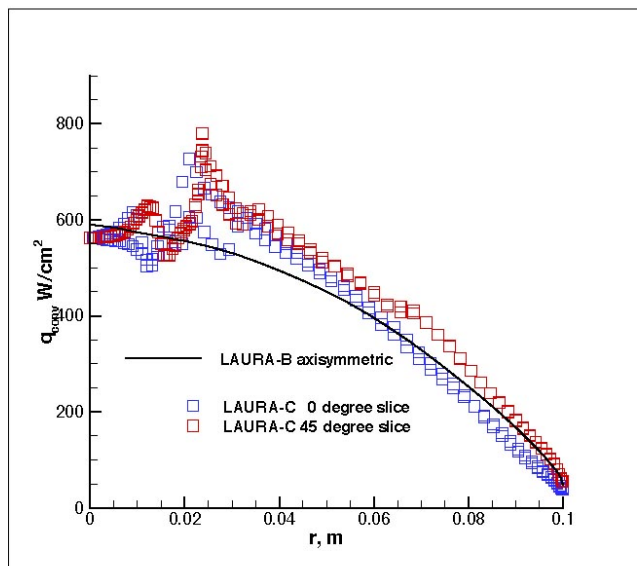

(d) Tet Grid, 1D reconstruction, Line Cuts at 0 and 45 degrees

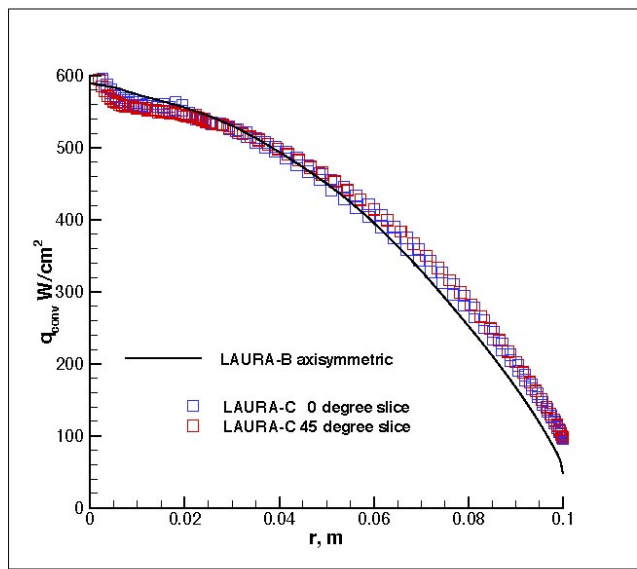

(f) Tet Grid, 3D reconstruction, Line Cuts at 0 and 45 degrees

Figure 9. Surface heating on sphere test problem. 


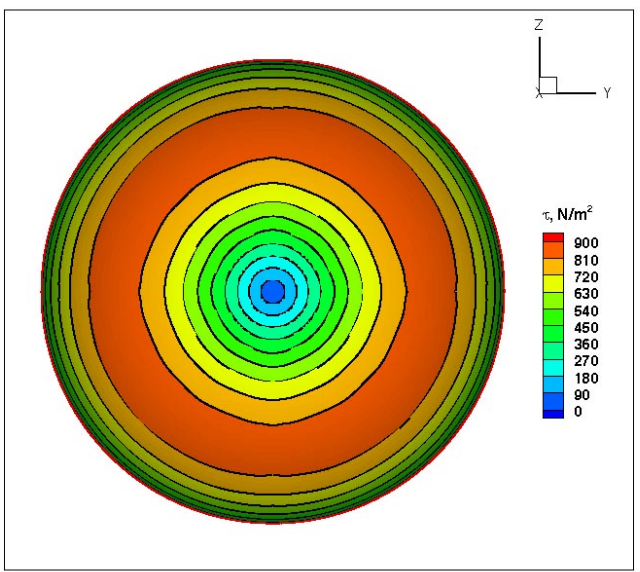

(a) Hex Grid, Surface Contours

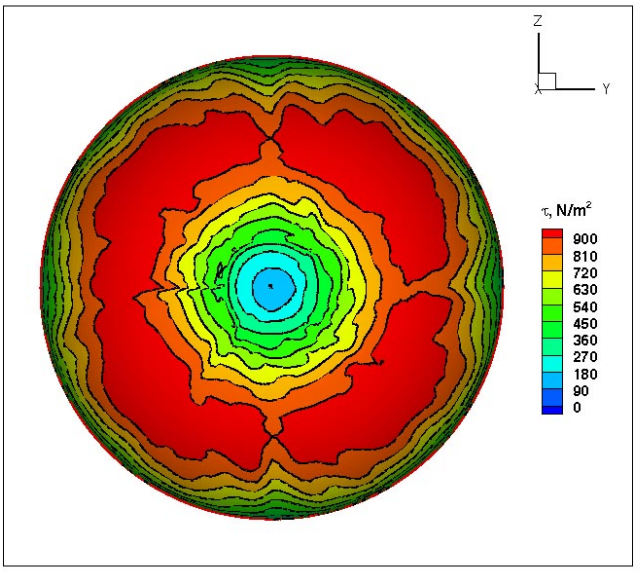

(c) Tet Grid, 1D reconstruction, Surface Contours

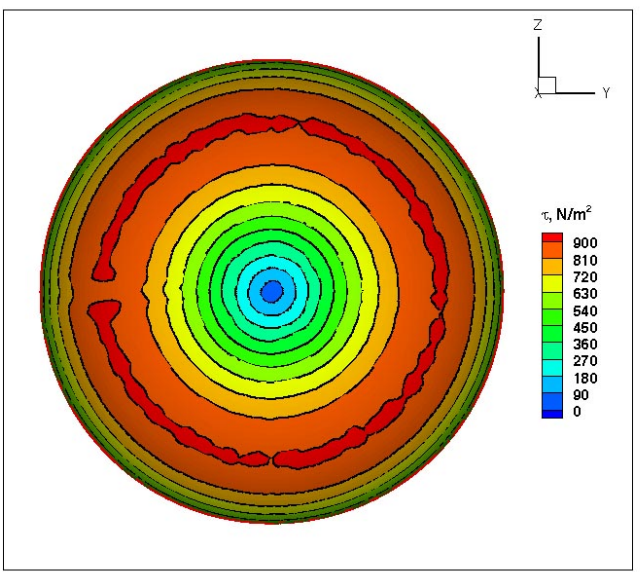

(e) Tet Grid, 3D reconstruction, Surface Contours

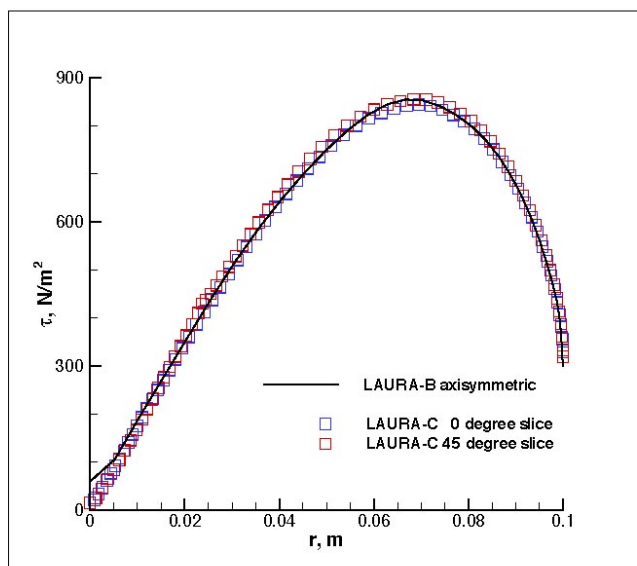

(b) Hex Grid, Line Cuts at 0 and 45 degrees

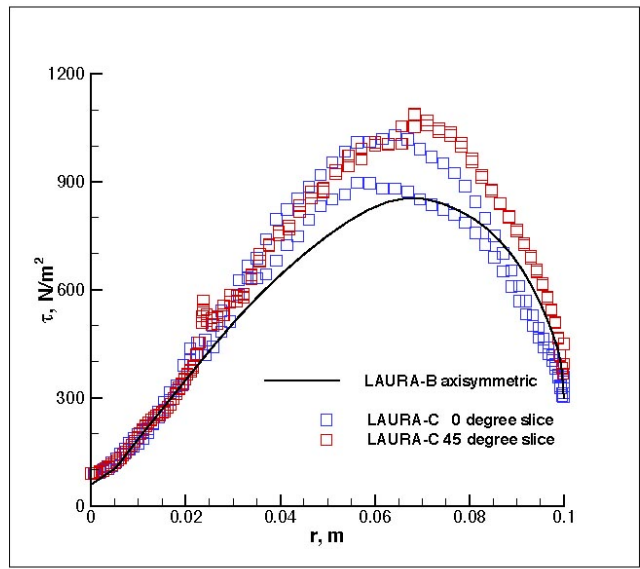

(d) Tet Grid, 1D reconstruction, Line Cuts at 0 and 45 degrees

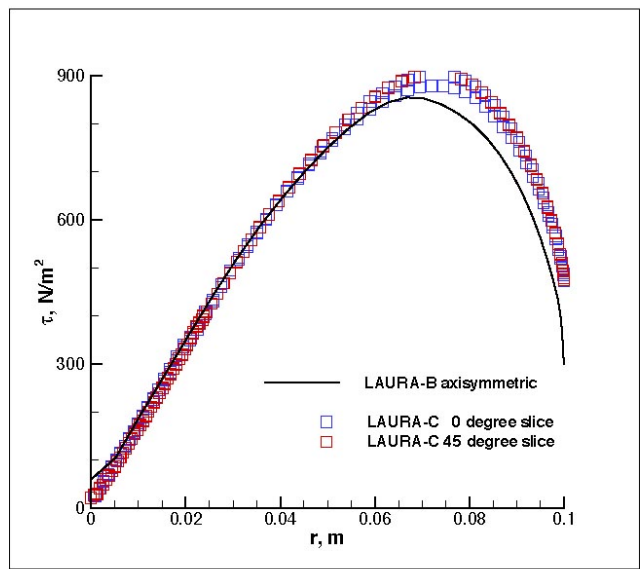

(f) Tet Grid, 3D reconstruction, Line Cuts at 0 and 45 degrees

Figure 10. Surface shear on sphere test problem. 


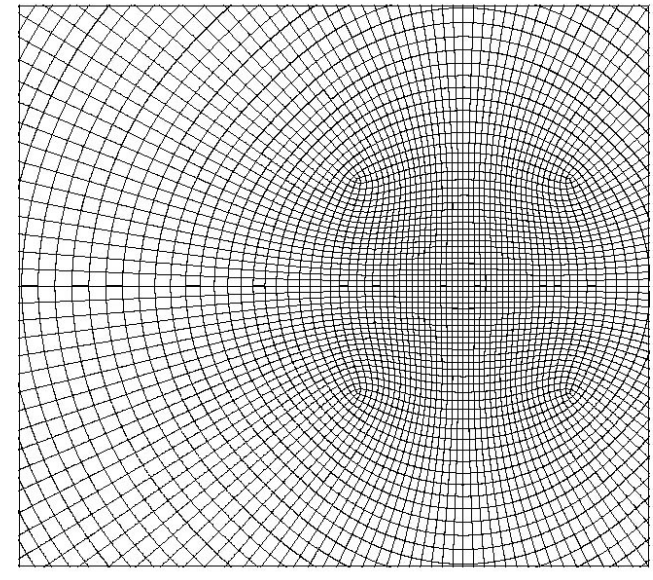

(a) Hex Grid

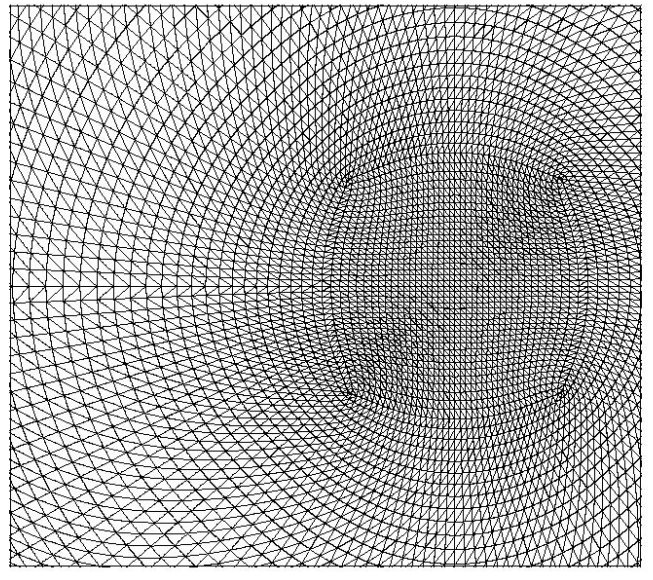

(b) Tet Grid

Figure 11. Surface grids on sphere test problem focused near stagnation point.

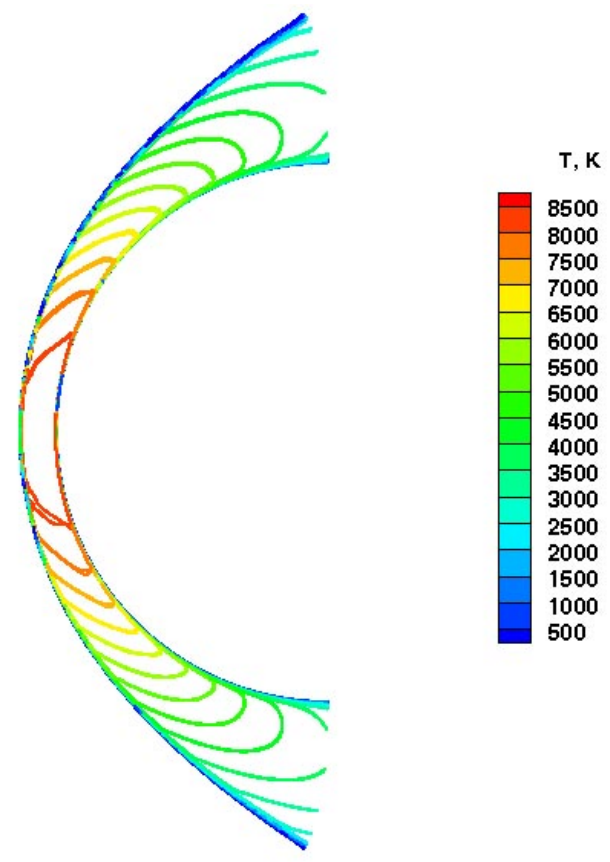

(a) Tet Grid, 1D reconstruction, Shock Layer Contours

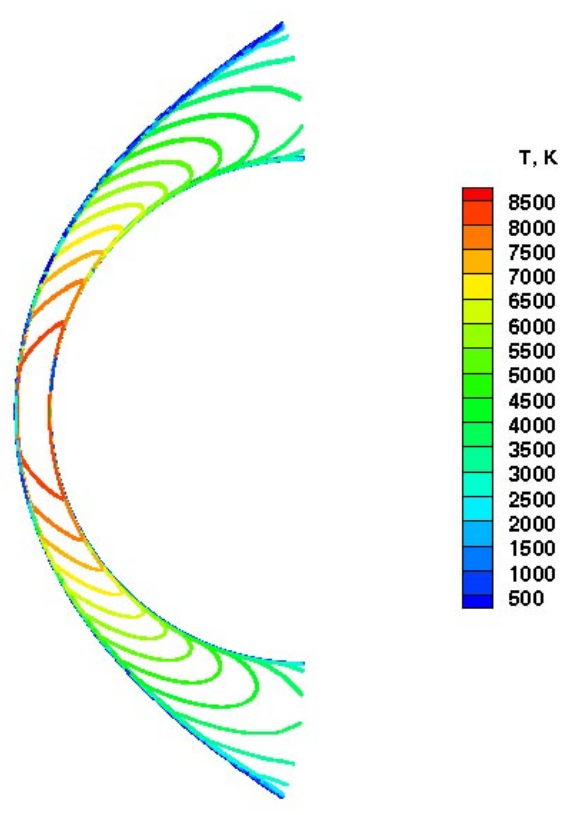

(b) Tet Grid, 3D reconstruction, Shock Layer Contours

Figure 12. Shock layer temperatures on slices at 0 and 45 degrees on sphere test problem.

\section{Summary of Numerical Results}

The option 1 results presented here requires three reconstructions per element whereas the baseline 1D algorithm requires one reconstruction per edge. The cylinder challenge problem with 10 spanwise cells required 14.78 seconds per relaxation step as compared to 6.84 seconds per step for the baseline 1D algorithm. Some improvement is expected when the inviscid and viscous terms are computed in a single loop over elements. This refactoring will eliminate some redundancies occurring in both the inviscid and viscous formulations. 
The baseline 1D algorithm provides good simulation of pressure but poor simulation of heating and shear, with as much as $\pm 20 \%$ dispersion about a symmetric distribution. The $3 \mathrm{D}$ reconstruction algorithm provides good simulation of pressure and eliminates almost all dispersion about a symmetric distribution on these biased grid test problems. The computed heating rates and shear stresses still show some differences with respect to the benchmark structured grid solutions. The comparisons with the sphere are better than the comparisons with the cylinder. The stagnation region heating for the sphere is within $4 \%$ of the benchmark. The stagnation region heating for the cylinder is within $8 \%$ of the benchmark. The peak shear on the sphere is within $8 \%$ of the benchmark. The peak shear on the cylinder is within $20 \%$ of the benchmark. There is no clear explanation why the sphere simulation is better than the cylinder simulation. The free stream conditions are different and the normal distribution of the original structured grids are different, with fewer nodes and greater stretching in the cylinder test problem. Certainly additional grid convergence tests are required here but the central point of this comparison is to evaluate the quality of an unstructured grid simulation relative to an equivalent (identical nodal distribution) structured grid distribution. In this regard it is evident that the 3D reconstruction (option 1) retains symmetry where expected in heating and shear on biased grids but does not necessarily attain a grid converged simulation with equivalent nodal distributions of a hexahedral grid system.

\section{Concluding Remarks}

The ultimate goal for any flow field simulation is to achieve a grid converged answer to a user specified target function (lift, drag, heat load) within a user specified uncertainty in a fully automated algorithm. Automated grid adaptation through localized enrichment, coarsening, and movement is a necessary component of such a simulation capability. The present work is motivated by a vision that an underlying unstructured grid system composed of simplex tetrahedra in three dimensions provides the greatest flexibility to adapt to shocks and shear layers in a hypersonic flow. However, it is recognized that hypersonic simulations on such a grid system produce poor quality heating and shear - associated with algorithms that are sensitive to element edges skewed to critical flow structures. The present paper introduces a three-dimensional flux reconstruction algorithm that is less dependent on edge orientation and produces improved results for heating and shear, even on highly biased, tetrahedral grid systems.

Two test problems have been investigated: a hypersonic flow over a three-dimensional cylinder, including resolution in the spanwise direction and hypersonic flow over a three-dimensional sphere. The tetrahedral cells used in the simulation are derived from a structured grid where cell faces are bisected across the diagonal resulting in a consistent pattern of diagonals running in a biased direction across the domain. This grid is known to accentuate problems in both shock capturing and stagnation region heating encountered with conventional, quasi-one-dimensional inviscid flux reconstruction algorithms. The test problem therefore provides a sensitive response to algorithm on heating.

The three-dimensional reconstruction shows significant improvement in symmetry (less than $3 \%$ dispersion) of heating with some penalty of ringing at the captured shock. While it retains symmetry where expected in heating and shear on biased grids it does not necessarily attain a grid converged simulation with the equivalent nodal distribution of a hexahedral grid system. Cost for inviscid reconstruction is almost a factor 3 more work than the baseline algorithm using equivalent storage requirements. The net penalty on the complete simulation is just over a factor of two in the cylinder test problem. Some cost can be offset by the ability to combine the inviscid and viscous formulations in the same loop to make best use of data in cache.

\section{References}

\footnotetext{
${ }^{1}$ Candler, G., Barnhardt, M., Drayna, T., Nompelis, I., Peterson, D., and Subbareddy, P., "Unstructured Grid Approaches for Accurate Aeroheating Simulations," AIAA Paper 2007-3959, June 2007.

${ }^{2}$ Gnoffo, P. A., "Simulation of Stagnation Region heating in Hypersonic Flow on tetrahedral Grids," AIAA Paper 20073960, June 2007.

${ }^{3}$ Cheatwood, F. M. and Gnoffo, P. A., "User's Manual for the Langley Aerothermodynamic Upwind Relaxation Algorithm (LAURA)," NASA TM 4674, April 1996.

${ }^{4}$ Gnoffo, P. A., Gupta, R. N., and Shinn, J. L., "Conservation Equations and Physical Models for Hypersonic Air Flows in Thermal and Chemical Nonequilibrium," NASA TP 2867, Feb. 1989.

${ }^{5}$ Wright, M. J., Candler, G. V., and Bose, D., "Data-parallel line relaxation method for the Navier-Stokes equations," AIAA J., Vol. 36, No. 9, 1998, pp. 1603-1609.
} 
${ }^{6}$ Holden, M. and Harvey, J., "Comparisons Between Experimental Measurements over Cone/Cone and Cylinder /Flare Configurations and Predictions Employing DSMC and Navier-Stokes Solvers," AIAA Paper 2001-1031, January 2001.

${ }^{7}$ Gnoffo, P. A., "CFD Validation Studies for Hypersonic Flow Prediction," AIAA Paper 2001-1025, Jan. 2001.

${ }^{8}$ Candler, G. V., Nompelis, I., Druguet, M.-C., Holden, M. S., Wadhams, T. P., Boyd, I. D., and Wang, W.-L., "CFD validation for hypersonic flight - Hypersonic double-cone flow simulations," AIAA Paper 2002-0581, January 2002.

${ }^{9}$ Nompelis, I., Drayna, T. W., and Candler, G. V., "Development of a Hybrid Unstructured Implicit Solver for the Simulation of Reacting Flows Over Complex Geometries," AIAA Paper 2004-2227, June 2004.

${ }^{10}$ Gnoffo, P. A., "Computational Fluid Dynamics Technology for Hypersonic Applications," AIAA Paper 2003-3259, July 2005.

${ }^{11}$ Paciorri, R. and Bonfiglioli, A., "Numerical Simulation of Shock-Shock Interations with an Unstructured Shock-Fitting Technique," Sixth european symposium on aerothermodynamics for space vehicles, Nov. 2008.

${ }^{12}$ Barter, G. E., "Shock Capturing with PDE-Based Artificial Viscosity for an Adaptive, Higher-Order Discontinuous Galerkin Finite Element Method," Mit dissertation, May 2008.

${ }^{13}$ Kontinos, D. A. and McRae, D. S., "Rotated Upwind Strategies for Solution of the Euler Equations," Tech. rep.

${ }^{14}$ Dadone, A. and Grossman, B., "Characteristic-Based, Rotated Upwind Scheme for the Euler Equations," AIAA J., Vol. 30, No. 9, 1992, pp. 2219-2226.

${ }^{15}$ Anderson, W. K. and Bonhaus, D. L., "An Implicit Upwind Algorithm for Computing Turbulent Flows on Unstructured Grids," Comp. and Fluids, Vol. 23, No. 1, Jan. 1994, pp. 1-21.

${ }^{16}$ Barth, T. J. and Jesperson, D. C., "The Design and Application of Upwind Schemes on Unstructured Meshes," AIAA Paper 89-0366, Jan. 1989.

${ }^{17}$ White, J. A. and Morrison, J. H., "A Pseudo-Temporal Multi-Grid Relaxation Scheme for Solving the Parabolized Navier-Stokes Equations," AIAA Paper 99-3360, June 1999.

${ }^{18}$ Gnoffo, P. A. and White, J. A., "Computational Aerothermodynamic Simulation Issues on Unstructured Grids," AIAA Paper 2004-2371, June 2004.

\section{Appendix A - Three-Dimensional Algorithm}

The flux components are defined using nodal weights based on the Green-Gauss formulation for derivatives in the component directions. Thus,

$$
\frac{\partial \mathbf{f}_{A}}{\partial x^{\prime}}=\frac{1}{\Omega_{A}} \sum_{k=\text { faces }} \overline{\mathbf{f}}_{k} A_{k} n_{k, x^{\prime}}
$$

where $\overline{\mathbf{f}}_{k}$ is the average value of $\mathbf{f}$ across all nodes defining surface $k$ of element $A$. Following in parallel the development of the two-dimensional algorithm and defining node 4 as the additional node required to define the tetrahedral element one obtains

$$
\begin{aligned}
\frac{\partial \mathbf{f}_{x^{\prime}, A}}{\partial x^{\prime}} & =\alpha_{3, x^{\prime}}\left(\mathbf{f}_{2, x^{\prime}}+\mathbf{f}_{1, x^{\prime}}+\mathbf{f}_{4, x^{\prime}}\right)+\alpha_{1, x^{\prime}}\left(\mathbf{f}_{3, x^{\prime}}+\mathbf{f}_{2, x^{\prime}}+\mathbf{f}_{4, x^{\prime}}\right) \\
& +\alpha_{2, x^{\prime}}\left(\mathbf{f}_{1, x^{\prime}}+\mathbf{f}_{3, x^{\prime}}+\mathbf{f}_{4, x^{\prime}}\right)+\alpha_{4, x^{\prime}}\left(\mathbf{f}_{1, x^{\prime}}+\mathbf{f}_{2, x^{\prime}}+\mathbf{f}_{3, x^{\prime}}\right)
\end{aligned}
$$

where

$$
\alpha_{k, x^{\prime}}=\frac{A_{k} n_{k, x^{\prime}}}{3 \Omega_{A}}
$$

and $k$ is a face index identified by the number of the opposite node. The sum $\sum_{k=\text { faces }} \alpha_{k, x^{\prime}}=0$ for any closed element. It is convenient to reorder and scale the coefficients as follows.

$$
\begin{aligned}
\frac{\partial \mathbf{f}_{A}}{\partial x^{\prime}} & =\left(\alpha_{2, x^{\prime}}+\alpha_{3, x^{\prime}}+\alpha_{4, x^{\prime}}\right) \mathbf{f}_{1}+\left(\alpha_{3, x^{\prime}}+\alpha_{1, x^{\prime}}+\alpha_{4, x^{\prime}}\right) \mathbf{f}_{2} \\
& +\left(\alpha_{1, x^{\prime}}+\alpha_{2, x^{\prime}}+\alpha_{4, x^{\prime}}\right) \mathbf{f}_{3}+\left(\alpha_{1, x^{\prime}}+\alpha_{2, x^{\prime}}+\alpha_{3, x^{\prime}}\right) \mathbf{f}_{4} \\
& \frac{\partial \mathbf{f}_{A}}{\partial x^{\prime}}=\frac{1}{\Delta x^{\prime}}\left[\beta_{1, x^{\prime}} \mathbf{f}_{1}+\beta_{2, x^{\prime}} \mathbf{f}_{2}+\beta_{3, x^{\prime}} \mathbf{f}_{3}+\beta_{4, x^{\prime}} \mathbf{f}_{4}\right]
\end{aligned}
$$

Note that $\Delta x^{\prime}$ can be interpreted as a distance across the element in $x^{\prime}$ direction. It is defined

$$
\frac{1}{\Delta x^{\prime}}=\sum_{n=\text { nodes }}\left(\left|\alpha_{n-1, x^{\prime}}+\alpha_{n+1, x^{\prime}}+\alpha_{n+2, x^{\prime}}\right|\right)
$$

where a cyclic indexing is assumed. The reordering and scaling yield the following relations for $\beta_{n, x^{\prime}}$.

$$
\begin{aligned}
\beta_{n, x^{\prime}} & =\Delta x^{\prime}\left(\alpha_{n-1, x^{\prime}}+\alpha_{n+1, x^{\prime}}+\alpha_{n+2, x^{\prime}}\right) \\
\sum_{n} \beta_{n, x^{\prime}} & =0 \\
\sum_{n}\left|\beta_{n, x^{\prime}}\right| & =1
\end{aligned}
$$




\section{A. Option 1 - Virtual Node Averaging in 3D}

The equations developed in the original section for two-dimensions are unchanged for three dimensions except that the sum over nodes includes a fourth node required to define a tetrahedral element.

\section{B. Option 2 - Weighted Average of Edges to Principal Node in 3D}

Select the largest of $\left|\beta_{n, x^{\prime}}\right|$ to identify the principal node for construction of $f_{x^{\prime}}$ and express it as a function of the remaining coefficients. Assume node 3 is the principal node. Then

$$
\begin{aligned}
d f_{x^{\prime}} & =\beta_{1, x^{\prime}} f_{1}+\beta_{2, x^{\prime}} f_{2}+\beta_{3, x^{\prime}} f_{3}+\beta_{4, x^{\prime}} f_{4} \\
& =\beta_{1, x^{\prime}} f_{1}+\beta_{2, x^{\prime}} f_{2}+\beta_{4, x^{\prime}} f_{4}-\left(\beta_{1, x^{\prime}}+\beta_{2, x^{\prime}}+\beta_{4, x^{\prime}}\right) f_{3} \\
& =\beta_{1, x^{\prime}}\left(f_{1}-f_{3}\right)+\beta_{2, x^{\prime}}\left(f_{2}-f_{3}\right)+\beta_{4, x^{\prime}}\left(f_{4}-f_{3}\right)
\end{aligned}
$$

The reconstructed flux in the direction $x^{\prime}, \mathbf{f}_{x^{\prime}}$, is computed as a weighted average of surrounding edges. Furthermore, it is noted that if any of the surrounding edges are parallel to $x^{\prime}$ then the weight of that edge will equal 1 and the weight of the other edges will equal zero.

$$
\begin{gathered}
\mathbf{f}_{x^{\prime}}=\left|\beta_{1, x^{\prime}}\right| \mathbf{f}_{1-3, x^{\prime}}+\left|\beta_{2, x^{\prime}}\right| \mathbf{f}_{2-3, x^{\prime}}+\left|\beta_{4, x^{\prime}}\right| \mathbf{f}_{4-3, x^{\prime}} \\
\mathbf{f}_{1-3, x^{\prime}}=\frac{1}{2}\left[\mathbf{f}_{1, x^{\prime}}+\mathbf{f}_{3, x^{\prime}}-\operatorname{sign}\left(\beta_{1, x^{\prime}}\right) \mathbf{R}_{1-3, x^{\prime}}^{-1}\left|\boldsymbol{\Lambda}_{1-3, x^{\prime}}\right|\left(d \tilde{\mathbf{q}}_{1-3, x^{\prime}}-d \tilde{\mathbf{q}}_{1-3, x^{\prime}, l i m}\right)\right] \\
\mathbf{f}_{2-3, x^{\prime}}=\frac{1}{2}\left[\mathbf{f}_{2, x^{\prime}}+\mathbf{f}_{3, x^{\prime}}-\operatorname{sign}\left(\beta_{2, x^{\prime}}\right) \mathbf{R}_{2-3, x^{\prime}}^{-1}\left|\boldsymbol{\Lambda}_{2-3, x^{\prime}}\right|\left(d \tilde{\mathbf{q}}_{2-3, x^{\prime}}-d \tilde{\mathbf{q}}_{2-3, x^{\prime}, l i m}\right)\right] \\
\mathbf{f}_{4-3, x^{\prime}}=\frac{1}{2}\left[\mathbf{f}_{4, x^{\prime}}+\mathbf{f}_{3, x^{\prime}}-\operatorname{sign}\left(\beta_{4, x^{\prime}}\right) \mathbf{R}_{4-3, x^{\prime}}^{-1}\left|\boldsymbol{\Lambda}_{4-3, x^{\prime}}\right|\left(d \tilde{\mathbf{q}}_{4-3, x^{\prime}}-d \tilde{\mathbf{q}}_{4-3, x^{\prime}, l i m}\right)\right]
\end{gathered}
$$

where

$$
\begin{aligned}
d \tilde{\mathbf{q}}_{1-3, x^{\prime}} & =\mathbf{R}_{1-3, x^{\prime}}\left(\mathbf{q}_{1}-\mathbf{q}_{3}\right) \\
d \tilde{\mathbf{q}}_{2-3, x^{\prime}} & =\mathbf{R}_{2-3, x^{\prime}}\left(\mathbf{q}_{2}-\mathbf{q}_{3}\right) \\
d \tilde{\mathbf{q}}_{4-3, x^{\prime}} & =\mathbf{R}_{4-3, x^{\prime}}\left(\mathbf{q}_{4}-\mathbf{q}_{3}\right) \\
d \tilde{\mathbf{q}}_{1-3, x^{\prime}, l i m} & =\operatorname{minmod}\left[2 d \tilde{\mathbf{q}}_{1, x^{\prime}}, 2 d \tilde{\mathbf{q}}_{1-3, x^{\prime}}, 2 d \tilde{\mathbf{q}}_{3, x^{\prime}}, \frac{1}{2}\left(d \tilde{\mathbf{q}}_{1, x^{\prime}}+d \tilde{\mathbf{q}}_{3, x^{\prime}}\right)\right] \\
d \tilde{\mathbf{q}}_{2-3, x^{\prime}, l i m} & =\operatorname{minmod}\left[2 d \tilde{\mathbf{q}}_{2, x^{\prime}}, 2 d \tilde{\mathbf{q}}_{2-3, x^{\prime}}, 2 d \tilde{\mathbf{q}}_{3, x^{\prime}}, \frac{1}{2}\left(d \tilde{\mathbf{q}}_{2, x^{\prime}}+d \tilde{\mathbf{q}}_{3, x^{\prime}}\right)\right] \\
d \tilde{\mathbf{q}}_{4-3, x^{\prime}, l i m} & =\operatorname{minmod}\left[2 d \tilde{\mathbf{q}}_{4, x^{\prime}}, 2 d \tilde{\mathbf{q}}_{4-3, x^{\prime}}, 2 d \tilde{\mathbf{q}}_{3, x^{\prime}}, \frac{1}{2}\left(d \tilde{\mathbf{q}}_{4, x^{\prime}}+d \tilde{\mathbf{q}}_{3, x^{\prime}}\right)\right] \\
d \tilde{\mathbf{q}}_{n, x^{\prime}} & =\Delta x^{\prime} \mathbf{R}_{n, x^{\prime}} \nabla \mathbf{q}_{n, L S q} \overrightarrow{\mathbf{n}}_{x^{\prime}}
\end{aligned}
$$

\section{Component Assembly on Faces in 3D}

The flux across each equivalent face $A n$ in $3 \mathrm{D}$ is constructed from the component flux in the principal direction and orthogonal to the principal direction.

$$
\mathbf{f}_{A n}=n_{A n, x^{\prime}} \mathbf{f}_{x^{\prime}}+n_{A n, y^{\prime}} \mathbf{f}_{y^{\prime}}+n_{A n, z^{\prime}} \mathbf{f}_{z^{\prime}}
$$

where $A n$ refers to face separating node $n$ from other nodes in the element. 